\title{
Editorial
}

Lifestyle
Genomics

\section{Cutting Edge Bioinformatics and Biostatistics Approaches Are Bringing Precision Medicine and Nutrition to a New Era}

\author{
Sanjeena Dang ${ }^{a}$ Nathalie Vialaneix ${ }^{b}$ \\ a Department of Mathematical Sciences, Binghamton University, Binghamton, NY, USA; ${ }^{\mathrm{b}}$ MIAT, INRA, \\ Université de Toulouse, Castanet-Tolosan, France
}

The traditional approach to medicine has relied on a population-based perspective for centuries. Decisions on treatment prescription were established according to an average response to a given therapy that aimed to treat a large number of patients with minimal overall side effects. Precision medicine is now viewed as an alternative, emerging and promising strategy to improve the efficacy of treatments. The United States' National Institute of Health defines precision medicine as "an emerging approach for disease treatment and prevention that considers individual variability in genes, environment, and lifestyle for each person" [1]. Although the term "precision medicine" has emerged recently, the idea has existed in medical practice for patient/donor matching since the mid-20th century for blood transfusion [2] and organ transplant [3]. Typically, matching is performed based on certain donor/patient attributes such as blood type, tissue type, size of the organ, etc. [4]. Precision medicine takes this concept to a broader level, whereby a more precise and personalized care plan is developed by incorporating insights from omics and analytics. The goal is to enable medical practitioners and researchers to accurately tailor care bearing in mind the differences between individuals that may lead to unique responses to different treatment regimens.

\section{KARGER}

(c) 2018 S. Karger AG, Basel

E-Mail karger@karger.com

www.karger.com/lfg
An omics approach for precision medicine became possible after the full human genome was first sequenced in April 2003 [5]. Targeted therapies are widely used for the treatment of different cancer subtypes by targeting and interfering with a specific molecule that is involved in the growth and progression of cancer [6]. For example, the targeted therapy to treat HER2-positive breast cancer has been approved by the Food and Drug Administration (FDA) since 1998 [7]. The number of human genomes sequenced has soared in the last decade, thanks to increasingly cheaper sequencing costs. A wide variety of cancer cells have been sequenced which has provided insight into novel fundamental genetic mechanisms involved in cancer initiation and progression [8]. This has enabled the identification of novel diagnostic biomarkers and potential targets for the development of other new targeted therapies [8]. Nowadays, in precision medicine, the use of various omics technologies beyond only genetic data is being increasingly recognized for driving improvements. Indeed, transcriptomics [9], epigenomics, metabolomics, metagenomics [10], or chromosome conformation signatures [11] have all played a successful role in providing new insights on how to improve the adaptation of therapies to patients. An increasing number of databases collect integrated personal omics $[12,13]$, and the

Sanjeena Dang

Department of Mathematical Sciences, Binghamton University

Binghamton, NY 13902 (USA)

E-Mail sdang@binghamton.edu 
volume of healthcare data is increasing exponentially. According to a report from EMC and International Data Corporation, the volume of healthcare data will increase from 153 exabytes ( 1 exabyte $=1$ billion gigabytes) in 2013 to 2,314 exabytes by 2020 [14]. Stephens et al. [15] provide an overview of the challenges that arise with the acquisition, storage, distribution, and analysis of these massive datasets.

However, the nature, volume, and complexity of such data are usually beyond the scope of direct interpretation or use by healthcare practitioners. There is a tremendous need and opportunity for data integration and analytic methods incorporating omics data in precision medicine. Data science has been called "the sexiest job of the 21st century" (to quote Thomas Davenport [16]). In precision medicine, it will likely be a key tool for major breakthroughs. Machine learning and artificial intelligence approaches are being increasingly utilized to identify individuals at risk, to predict which prevention strategies work best on patients, to automatically screen different subtypes of diseases, or to perform drug repurposing. They have also been successfully implemented for the diagnosis and prognosis of diseases. For example, Esteva et al. [17] have recently shown that an automated classification algorithm based on deep convolutional neural networks that only used image and disease labels as inputs achieved near human expert performance in identifying different types of skin cancers. Another successful application of deep learning techniques was for automatic detection of diabetic retinopathy and diabetic macular edema in retinal fundus photographs through image data analysis [18]. The Stanford Byers Center for Biodesign is developing predictive tools for potential heart transplant rejection and early detection of pediatric asthma using a monitoring device [19]. Investment in the development of efficient data mining algorithms and the application to health data are burgeoning. In the fiscal year 2016, USD 215 million were devoted by President Obama's Precision Medicine Initiative [20] to support research in data acquisition and interoperability and scale up efforts to identify genomic drivers in cancer. Another initiative in 2016 was the USD 600 million Chan Zuckerberg BioHub, a collaborative project between Stanford, UC San Francisco, and UC Berkeley that aimed to bring together expert scientists from various fields and apply some of the most advanced technologies (existing and new) to solve some of the world's biggest health problems [21].

However, many challenges remain. To disseminate these approaches more widely in precision medicine, the fields of bioinformatics and biostatistics face several challenges. Not only are datasets massive, which necessitates efficient and scalable methods to handle them, but they are also often high dimensional, i.e., the number of features to analyze being far larger than the number of individuals on which the data have been acquired. This can be further complicated if multivariate measurements are taken over time. Given population heterogeneity and the large number of confounding factors - especially for epidemiologic studies - small effects may be hard to detect due to reduced statistical power or difficulty to handle hidden subpopulation characteristics [22] that might affect the study conclusions. In addition, data are often very heterogeneous, coming in various formats, scales, and types. Data interoperability, multi-scale aggregation, and multi-type methods are thus needed for efficient integration of omics data. To address these issues, a large number of methods, using tools from statistics, machine learning, and artificial intelligence are currently being developed and investigated for omics data analysis [13, 23-25]. From a methodological point of view, data analysis methods can be distinguished by at least three important characteristics.

(1) The purpose of the integration is either an exploratory analysis (i.e., unsupervised approaches) or predictive analysis (i.e., supervised approaches) or even semisupervised approaches that fall between supervised and unsupervised approaches. Unsupervised approaches can help stratify the population of patients into subtypes (iCluster [26] or PARADIGM [27], for instance), and supervised approaches have yielded interesting insight into drug-disease association prediction (DDR [28]), for example. When part of the data is labelled, utilizing that information in a semi-supervised framework has been shown to greatly improve classification performance [29].

(2) The method can handle integration of specific omics data (transcriptomics and copy number variation [30]) or is made generic, for example, by using a graph- or kernel-based representation (mixKernel [31]) or relevant transformations combined with a generic integration method (mixOmics [32]).

(3) The way the method handles the integration of multiple omics, for instance, as described by Mutch et al. [22], by simply concatenating the (almost necessarily numerical) data into one big dataset prior to an analysis, by performing separate analyses for all data that are combined afterwards or by transforming the data into a common object type (graph or kernel) that can easily be combined in a way that is optimal for a target analysis.
Dang/Vialaneix 
Moreover, in parallel to the improvement of statistical or learning methods for precision medicine, an ongoing challenge has emerged. Specifically, providing a better understanding of these complex algorithms and recommendations made by machines is critical to make them more widely adopted by the medical community as reliable tools to aid in decision-making. Also, looking to the future, data sharing and integration of heterogeneous data sources will play a big part in precision medicine. Explosion of technologies, such as smartphones and smart watches, is also providing a novel and massive influx of low-cost universal individual-level health surveillance data, such as monitoring physical activity, dietary habits, and several health outcomes (e.g., heart rate), among other things. Developing and applying tools to incorporate multisource massive data efficiently in medical decision-making will take personal healthcare to a new level. This also triggers the need to establish standards and universal guidelines to facilitate data sharing, such as the MIAME initiative for microarray [33].

Finally, precision medicine goes beyond individualized treatment plans. From a holistic perspective, it involves the development of screening, diagnosis, and preventative strategies. Lifestyle plays a big role in determining optimal health and well-being. Dietary choices have a crucial effect on the prevention of age-related diseases, and the risks and benefits of these dietary choices vary from individual to individual. Indeed, nutrigenomics, which aims to understand the interaction between genes and food and how it influences human health, is gaining increasing attention. Although cancer has been the primary focus of precision medicine so far, nutrigenomics provides an opportunity for precision healthcare on a larger scale. Prevention, management and treatment strategies based on an individual's dietary habits, lifestyle choices, and genetics for complex diseases, such as diabetes and cardiovascular diseases, are emerging [34, 35]. The focus of precision medicine is evolving from solely optimizing treatment to including the development of individualized prevention and management plans for disease prevention [36]. Development and application of emerging and current biostatistics and bioinformatics approaches will play a central role in precision medicine and nutrigenomics. Thus, the scope of Lifestyle Genomics includes the publication of exploratory and predictive approaches to support the improvement of precision medicine and nutrition to better understand the complex relationships between lifestyle factors and genes. Therefore, we invite methodologists and researchers developing and applying such methods and tools to consider Lifestyle Genomics as a journal to disseminate their research.

\section{Disclosure Statement}

The authors have no conflicts of interest to disclose. However, both authors are members of the Editorial Board for Lifestyle Genomics.

\section{References}

1 National Library of Medicine [Internet]. What is precision medicine? [cited June 9, 2018]. Available from: https://ghr.nlm.nih. gov/primer/precisionmedicine/definition.

2 Dance A. Medical histories. Nature. 2016 Sep; 537(7619):S52-3.

3 Goldman KE. Dental management of patients with bone marrow and solid organ transplantation. Dent Clin North Am. 2006 Oct;50(4): 659-76.

4 U.S. Department of Health \& Human Services [Internet]. Donor Matching System [cited August 10, 2018]. Available from: https:// optn.transplant.hrsa.gov/learn/abouttransplantation/donor-matching-system/.

5 National Human Genome Research Institute. International Consortium Completes Human Genome Project [News Release] 2003, April 14 [cited July 17, 2018]. Available from: https://www.genome.gov/11006929/.
6 National Cancer Institute [Internet]. Targeted cancer therapies [cited September 23, 2018]. Available from: https://www.cancer. gov/about-cancer/treatment/types/targetedtherapies/targeted-therapies-fact-sheet.

7 Lewis Phillips GD, Li G, Dugger DL, Crocker LM, Parsons KL, Mai E, et al. Targeting HER2positive breast cancer with trastuzumabDM1, an antibody-cytotoxic drug conjugate. Cancer Res. 2008 Nov;68(22):9280-90.

8 Wang L, Wheeler DA. Genomic sequencing for cancer diagnosis and therapy. Annu Rev Med. 2014;65(1):33-48.

9 Das K, Chan XB, Epstein D, Teh BT, Kim KM, Kim ST, et al. NanoString expression profiling identifies candidate biomarkers of RAD001 response in metastatic gastric cancer. ESMO Open. 2016 Feb;1(1):e000009.

10 Viaud S, Saccheri F, Mignot G, Yamazaki T, Daillère $R$, Hannani $D$, et al. The intestinal microbiota modulates the anticancer immune effects of cyclophosphamide. Science. 2013 Nov;342(6161):971-6.
11 Carini C, Hunter E. Scottish Early Rheumatoid Arthritis Inception cohort Investigators, Ramadass AS, Green J, Akoulitchev A, McInnes IB, Goodyear CS. Chromosome conformation signatures define predictive biomarkers of inadequate response to methotrexate in early rheumatoid arthritis. J Transl Med. 2018 Jan;16(1):18.

12 Chen R, Mias GI, Li-Pook-Than J, Jiang L, Lam HY, Chen R, et al. Personal omics profiling reveals dynamic molecular and medical phenotypes. Cell. 2012 Mar;148(6):1293-307.

13 Gligorijević V, Malod-Dognin N, Pržulj N. Integrative methods for analyzing big data in precision medicine. Proteomics. 2016 Mar; 16(5):741-58.

14 EMC. IDC. The Digital Universe: Driving Data Growth in Healthcare. 2014. [cited June 9, 2018]. Available from: http://www.emc. com/analyst-report/digital-universe-healthcare-vertical-report-ar.pdf.
Bioinformatics and Biostatistics in Precision Medicine and Nutrition
Lifestyle Genomics 2018;11:73-76 DOI: $10.1159 / 000494131$ 
15 Stephens ZD, Lee SY, Faghri F, Campbell RH, Zhai C, Efron MJ, et al. Big Data: astronomical or Genomical? PLoS Biol. 2015 Jul; 13(7):e1002195.

16 Davenport T. Data Scientist: The Sexiest Job of the 21st Century. 2012 [cited June 18, 2018]. Available from: https://www.youtube. com/watch? $v=z Q \operatorname{lnbrHgzCE}$.

17 Esteva A, Kuprel B, Novoa RA, Ko J, Swetter SM, Blau HM, et al. Dermatologist-level classification of skin cancer with deep neural networks. Nature. 2017 Feb;542(7639):115-8.

18 Gulshan V, Peng L, Coram M, Stumpe MC, $\mathrm{Wu}$ D, Narayanaswamy A, et al. Development and validation of a deep learning algorithm for detection of diabetic retinopathy in retinal fundus photographs. JAMA. 2016 Dec; 316(22):2402-10

19 Medicine S. Harnessing the Power of Data in Health 2017 [cited June 9, 2018]. Available from: https://med.stanford.edu/content/ dam/sm/sm-news/documents/StanfordMedicineHealthTrendsWhitePaper2017.pdf.

20 United States, Office of the Press Secretary, FACT SHEET: President Obama's Precision Medicine Initiative, The White House, 2015, Jan 30 [cited June 9, 2018]. Available from: https://obamawhitehouse.archives.gov/thepress-office/2015/01/30/fact-sheet-president-obama-s-precision-medicine-initiative.

21 Berkeley UC. UC Berkeley to partner in $\$ 600 \mathrm{M}$ Chan Zuckerberg science 'Biohub' [press release]. 2016, Sept 21. [cited June 9, 2018]. Available from: http://news.berkeley. edu/2016/09/21/biohub/.
22 Mutch DM, Zulyniak MA, Rudkowska I, Tejero ME. Lifestyle genomics: addressing the multifactorial nature of personalized health. Lifestyle Genom. 2018;11(1):1-8.

23 Kristensen VN, Lingjærde OC, Russnes HG, Vollan HK, Frigessi A, Børresen-Dale AL. Principles and methods of integrative genomic analyses in cancer. Nat Rev Cancer. 2014 May;14(5):299-313.

24 Ritchie MD, Holzinger ER, Li R, Pendergrass SA, Kim D. Methods of integrating data to uncover genotype-phenotype interactions. Nat Rev Genet. 2015 Feb;16(2):85-97.

25 Bersanelli M, Mosca E, Remondini D, Giampieri E, Sala C, Castellani G, et al. Methods for the integration of multi-omics data: mathematical aspects. BMC Bioinformatics. 2016 Jan;17(S2 Suppl 2):15.

26 Shen R, Olshen AB, Ladanyi M. Integrative clustering of multiple genomic data types using a joint latent variable model with application to breast and lung cancer subtype analysis. Bioinformatics. 2009 Nov;25(22):290612.

27 Vaske CJ, Benz SC, Sanborn JZ, Earl D, Szeto $\mathrm{C}$, Zhu J, et al. Inference of patient-specific pathway activities from multi-dimensional cancer genomics data using PARADIGM. Bioinformatics. 2010 Jun;26(12):i237-45.
28 Zhang P, Wang F, Hu J. Towards drug repositioning: a unified computational framework for integrating multiple aspects of drug similarity and disease similarity. AMIA Annu Symp Proc. 2014 Nov;2014:1258-67.

29 Shi M, Zhang B. Semi-supervised learning improves gene expression-based prediction of cancer recurrence. Bioinformatics. 2011 Nov;27(21):3017-23.

30 Akavia UD, Litvin O, Kim J, Sanchez-Garcia F, Kotliar D, Causton HC, et al. An integrated approach to uncover drivers of cancer. Cell. 2010 Dec;143(6):1005-17.

31 Mariette J, Villa-Vialaneix N. Unsupervised multiple kernel learning for heterogeneous data integration. Bioinformatics. 2018 Mar; 34(6):1009-15.

32 Rohart F, Gautier B, Singh A, Lê Cao KA. mixOmics: an R package for 'omics feature selection and multiple data integration. PLOS Comput Biol. 2017 Nov;13(11):e1005752.

33 Functional Genomics Data Society [Internet]. MAIME Minimum Information About a Microarray Experiment [cited September 23, 2018]. Available from: http://www.fged.org/ projects/miame/.

34 Florez JC. Precision medicine in diabetes: is it time? Diabetes Care. 2016 Jul;39(7):1085-8

35 Leopold JA, Loscalzo J. Emerging role of precision medicine in cardiovascular disease. Circ Res. 2018 Apr;122(9):1302-15.

36 Jeejeebhoy K, Dhaliwal R, Heyland DK, Leung R, Day AG, Brauer P, et al. Family physician-led, team-based, lifestyle intervention in patients with metabolic syndrome: results of a multicentre feasibility project. CMAJ Open. 2017 Mar;5(1):E229-36. 